\section{Impacto da sintomatologia osteomuscular na qualidade de vida de professores}

\section{Impact of osteomuscular symptoms on the quality of life of teachers}

\section{Marcos Henrique Fernandes ${ }^{\prime}$ \\ Vera Maria da Rocha"}

Ana Angelica Ribeiro Fagundes"'I

' Programa de Pós-graduação em Enfermagem e Saúde da Universidade Estadual do Sudoeste da Bahia; Programa de Ciências da Saúde da Universidade Federal do Rio Grande do Norte.

"Universidade Federal do Rio Grande do Sul; Programa de Pós-Graduação em Ciências da Saúde da Universidade Federal do Rio Grande do Norte.

II' Universidade Estadual do Sudoeste da Bahia.

\section{Resumo}

O presente estudo objetivou verificar o impacto da sintomatologia osteomuscular na qualidade de vida dos professores da rede municipal de ensino de Natal/RN. Trata-se de uma pesquisa descritiva-analítica com corte transversal, que teve como amostra 242 docentes da rede básica. Foi utilizado o Whoqol-breve para avaliação da qualidade de vida e o questionário Nórdico para os sintomas osteomusculares. Na análise dos dados foram utilizados procedimentos da estatística descritiva e o teste de MannWhitney, com um nível de significância de $5 \%$. A prevalência da referida sintomatologia foi de $63,2 \%$ entre os investigados, levando-se em consideração os sete dias anteriores ao momento da coleta dos dados. Todos os domínios da qualidade de vida apresentaram-se comprometidos entre o grupo de docentes que apresentaram os sintomas osteomusculares quando comparados ao grupo sem a sintomatologia, sendo encontrado no domínio Físico valor de $p<0,001$, no Psicológico $p<0,001$, Relação Social $p<0,001$ e Meio Ambiente $p<0,001$. Dessa forma, a presença de sintomatologia osteomuscular apresentou grande correlação estatística com o comprometimento da qualidade de vida dos professores da rede básica do município de Natal, RN.

Palavras-chave: Qualidade de vida. Docentes. Saúde do trabalhador. Transtornos traumáticos cumulativos. Educação. Dor. 


\section{Abstract}

The aim of this study was to determine the impact of osteomuscular symptoms on the quality of life of public school teachers in Natal, Brazil. This is a descriptive-analytical study with a cross-sectional design, comprising a sample of 242 teachers. The WHOQOL-brief was used to assess quality of life and the Nordic questionnaire for osteomuscular symptoms. Descriptive statistical procedures and Mann-Whitney test were used in data analysis, with the significance level set at $5 \%$. The prevalence of the aforementioned symptoms among the subjects investigated was $63.2 \%$, considering the seven days before data collection. All quality of life domains were compromised in the group of teachers who presented osteomuscular symptoms, when compared to the asymptomatic group. The physical, psychological, social and environmental domains all had p-values smaller than 0.001 . Thus, the presence of osteomuscular symptoms showed a strong statistical correlation with lower quality of life of teachers from the basic education system of Natal, Brazil.

Keywords: Quality of life. Teachers. Worker's health. Cumulative trauma disorders. Education. Pain.

\section{Introdução}

O processo de globalização mundial está fortemente associado ao desenvolvimento industrial e às profundas alterações nas condições e precarização do trabalho, as quais foram marcadas por consequências como o aumento da jornada de trabalho e o acúmulo de funções, além de uma maior exposição a fatores de riscos para saúde, o que contribuiu decisivamente para deterioração das condições de saúde dos trabalhadores ${ }^{1,2}$.

No âmbito dessas transformações ocorridas no mundo do trabalho, a escola também foi submetida a diversas mudanças que abrangeram toda a sua estrutura e as formas de organização de trabalho, passando a atividade docente a ser orientada pelo modelo de produção capitalista, o que provocou efeitos perversos de depreciação e desqualificação social, psicológica e biológica dos professores ${ }^{3,4}$.

A complexidade e o ritmo acelerado do trabalho dos professores impõem um processo de esforço permanente a esses profissionais, os quais são considerados uma das categorias ocupacionais que mais tem sofrido agravos à saúde. Fatores como baixos salários, burocratização, hierarquização das relações de trabalho, jornada de trabalho tripla, além da carência de recursos materiais e humanos são elementos importantes para o surgimento do referido quadro $^{5}$.

Nesse contexto, o trabalho docente extrapolou os limites das atividades desenvolvidas em sala de aula, passando a incorporar também diversas atividades como elaboração de projetos, discussão coletiva do currículo, assessoramento psicológico, educação para o trânsito, construção de hábitos de saúde, dentre outras, sendo esse profissional responsabilizado pelo sucesso ou o fracasso dos processos educacionais ${ }^{3,6}$.

Emerge dessa situação um cenário com efeitos adversos, proporcionando aos docentes um conjunto de doenças de natureza psicossomática e física, as quais exercem uma forte influência na qualidade de vida destes profissionais ${ }^{3}$. 
Através de diferentes perspectivas, a expressão Qualidade de Vida (QV) está diretamente associada à saúde, tendo sido aproximada ao grau de satisfação do individuo em sua vida amorosa, familiar, social e ambiental, podendo abranger diversos significados construídos socialmente em diferentes espaços e histórias ${ }^{7,8}$. Foi definida de uma forma mais genérica como a percepção do individuo de sua posição na vida no contexto da cultura e sistema de valores, nos quais ele vive em relação aos seus objetivos, expectativas, padrões e preocupações ${ }^{\text {. }}$.

Portanto, os riscos ocupacionais provenientes das condições de trabalho dos professores podem provocar mecanismos desencadeadores e agravadores de morbidades relacionadas direta ou indiretamente ao trabalho, como os sintomas osteomusculares $^{1,2}$. O referido quadro patológico afeta diversas categorias profissionais e apresenta uma considerável relevância social devido à sua abrangência e magnitude ${ }^{2}$.

Os distúrbios decorrentes do sistema musculoesquelético podem levar ao aparecimento de diversos sinais como a dor e incapacidade funcional, provocando repercussões importantes sobre a qualidade de vida dos indivíduos acometidos ${ }^{10}$. Entre a classe trabalhadora docente é bastante comum a existência de doenças do sistema osteomuscular, as quais se constituem numa das principais causas de absenteísmo das atividades de trabalho ${ }^{11-13}$.

O quadro descrito possui uma origem multifatorial complexa, como exigências mecânicas repetidas por longos períodos de tempo, fatores ligados à organização do trabalho, como a busca por produtividade, além de características individuais e do estilo de vida do individuo ${ }^{14}$.

Há poucos estudos realizados na população brasileira que versem sobre o impacto da sintomatologia osteomuscular na qualidade de vida de professores, objetivo que motivou a realização do presente estudo com os docentes da rede municipal de ensino da cidade de Natal, RN. Os resultados apresentados neste estudo poderão contribuir para a elaboração de políticas públicas que visem à promoção de saúde no contexto do trabalho docente.

\section{Métodos}

Este estudo é parte de um projeto maior intitulado "Características sociodemográficas, ocupacionais e de saúde na avaliação da qualidade de vida de professores de rede municipal de Natal/RN", sendo caracterizado como descritivo-analítico e de corte transversal, não possuindo nenhum tipo de conflito de interesse.

A população foi constituída pelos professores da educação básica (ensino infantil e fundamental) da rede municipal de Natal/ $\mathrm{RN}$, que segundo dados da Secretaria Municipal de Educação perfaziam um contingente de 2.651 indivíduos, no ano de 2007.

Ao total foram selecionados 264 docentes, tendo ocorrido a perda de 22 indivíduos que não se dispuseram ou não entregaram os questionários da coleta dos dados. Com isso o estudo foi composto por uma amostra de 242 professores, que foi calculada a partir da população referida e de uma referência de média para os domínios da qualidade de vida de $56,15 \%{ }^{15}$, além do nível de confiança de $95 \%$ e margem de erro de $3 \%$.

Inicialmente foi determinado o número de indivíduos a serem selecionados em cada uma das quatro zonas distritais da cidade, respeitando a proporcionalidade entre $o$ número total de docentes em cada zona distrital como o número total da amostra. Posteriormente os professores foram escolhidos de maneira aleatória por meio de sorteio simples a partir da listagem numerada dos mesmos, sendo excluídos antes do sorteio aqueles que ocupavam cargos administrativos e que estavam afastados da atividade docente no momento da coleta. Após esclarecimento sobre os objetivos do estudo, foi entregue aos professores participantes um questionário não identificado composto por três partes, que foi recolhido num prazo máximo de duas semanas na escola de trabalho. Esta coleta de dados foi realizada no último bimestre letivo do ano de 2007. 
A primeira parte do questionário foi constituída por questões referentes a dados sociodemográficos (sexo, idade, estado civil, renda e nível de escolaridade) e dados ocupacionais (tempo de trabalho na docência, carga horária semanal de trabalho, número de alunos em sala de aula e se a escola é localizada no mesmo bairro da residência do professor). É importante ressaltar que a carga horária semanal relatada pelos investigados no presente estudo se refere apenas às atividades exercidas nas escolas.

A segunda parte correspondia à avaliação da qualidade de vida, sendo utilizado o instrumento World Health Organization Quality Of Life/Bref (WHOQOL/breve), validado para a população brasileira, apresentando consistência interna satisfatória (Coeficiente de Cronbach variando entre $0,69$ a 0,91$)^{16}$.

OWHOQOL/breve é um instrumento de avaliação constituído por 26 questões, que aborda quatro domínios que expressam a qualidade de vida dos indivíduos investigados: físico (fadiga, dependência de medicação ou tratamento, energia, realização de atividades da vida diária, capacidade de trabalho e mobilidade), psicológico (sentimentos positivos, espiritualidade, pensar, memória, concentração, aprender, imagem corporal, auto-estima, sentimentos negativos), meio ambiente (segurança física, ambiente físico, poluição, recursos financeiros, recreação/lazer, moradia e transporte) e relações sociais (satisfação com as relações pessoais, atividade sexual e apoio dos amigos).

Para avaliar os resultados foi dado um escore para cada questão, variando de um a cinco, com o objetivo de transformar os resultados de cada domínio em uma escala graduada de 0 a 100 , sendo que o zero (0) corresponde a um pior estado de saúde e cem (100) a um melhor estado, possibilitando a análise individual de cada dimensão.

Foi utilizado na terceira parte, o questionário nórdico (Nordic Musculoskeletal Questionnaire), para avaliação dos sintomas osteomusculares, o qual foi validado e adaptado culturalmente à língua portuguesa ${ }^{17}$.
O instrumento é composto por uma figura do corpo humano, mostrando as regiões anatômicas dos membros (pescoço, ombros, parte superior e inferior das costas, cotovelos, punhos/mãos, quadril/coxas, joelhos e tornozelos/pés). Nessa parte, o individuo respondia com um X (xis) em cada questão as respostas "sim" ou "não", em relação à ocorrência de sintomas como dor, formigamento/dormência nos últimos 12 (doze) meses e nos últimos 7 (sete) dias.

O Projeto de Pesquisa foi aprovado pelo Comitê de Ética em Pesquisa da Universidade Federal do Rio Grande do Norte, obedecendo às normas da Resolução 196/96, incluindo o termo de consentimento livre e esclarecido.

A análise dos resultados foi realizada através do programa SPSS - "Statistical Package for the Social Sciences” - versão 15.0, sendo verificada a distribuição dos dados por meio do teste de Kolmogorov - Smirnov, o qual apontou que os mesmos não seguem os princípios da normalidade. Foi utilizada a estatística descritiva, com determinação das médias (x) e desvio-padrão (DP) para as variáveis quantitativas, frequência simples e relativa para as variáveis categóricas.

O teste de Mann-Whitney, que é usado para comparação de dois grupos, verificou a existência de diferenças nos resultados dos domínios da qualidade de vida entre os professores com e sem a presença da sintomatologia osteomuscular, sendo adotado como critério para inclusão do grupo de professores com sintomas osteomusculares aqueles que marcaram sim em qualquer região anatômica do corpo nos últimos sete dias do período da realização do estudo, para que fosse evitado o viés de memória. Foi adotado um nível de significância de $5 \%$.

\section{Resultados}

A maioria dos professores investigados $81,7 \%$ era do sexo feminino e $18,3 \%$ do sexo masculino. A média de idade foi de 43,5 anos e, com relação ao estado civil, $62 \%$ dos docentes eram casados. 
Quanto à escolaridade dos indivíduos estudados, a maioria, $53,4 \%$, apresentava apenas nível superior completo e $42,4 \%$ possuíam também pós-graduação. A renda média mensal dos professores era de $\mathrm{R} \$$ $1.475,30$ correspondente a aproximadamente quatro salários mínimos na época da coleta dos dados.

O tempo médio de exercício da atividade docente relatado foi de 18,25 anos. A carga horária semanal de trabalho apresentou média de 32 horas e a média de alunos por sala de aula foi de 32. A maior parte dos professores $(64,9 \%)$ não residia no mesmo bairro em que estão localizadas as escolas de trabalho. Na Tabela 1 estão descritos os resultados da análise descritiva das variáveis quantitativas.
A prevalência da sintomatologia osteomuscular no último ano, independente da região corporal afetada, foi de $93 \%$ na amostra de professores da rede municipal de ensino de Natal, RN estudada. As regiões corporais em que se registraram mais queixas foram a parte superior das costas com $58,7 \%$, seguida da parte inferior das costas, com $53,7 \% \%$, e pescoço com $53,7 \%$ (Tabela 2 ).

Quanto ao impedimento de realizar atividades devido a tais sintomas nos últimos 12 meses, $47,7 \%$ professores responderam positivamente. $51,5 \%$ dos investigados relataram ter realizado alguma consulta a profissional da saúde devido ao referido problema.

A ocorrência da sintomatologia osteomuscular nos últimos sete dias, levando em

Tabela 1 - Distribuição dos resultados da análise descritiva das variáveis quantitativas dos professores da rede municipal de ensino de Natal, RN, 2007.

Table 1 - Distribution of results of the quantitative descriptive analysis of teachers from the public school system of Natal, RN, Brazil, 2007.

\begin{tabular}{lcccccc}
\hline Variável & $\mathrm{n}$ & $\overline{\mathrm{X}}$ & Mediana & $\mathrm{DP}$ & $\mathrm{IC}(95 \%)$ & $\mathrm{Q} 25-\mathrm{Q} 75$ \\
\hline Idade (anos) & 239 & 43,50 & 42,0 & 9,54 & $42,28-44,71$ & $37,0-50,0$ \\
Renda (R\$) & 213 & $1.475,3$ & $1.385,0$ & 663,55 & $1.385,7-1.564,9$ & $943,0-1.815,0$ \\
Tempo de Trabalho (anos) & 240 & 18,25 & 18,0 & 25 & $17,08-19,43$ & $11,0-25,0$ \\
Carga Horária Semanal(h) & 231 & 31,73 & 28,0 & 14,62 & $29,84-33,63$ & $20,0-40,0$ \\
Média de alunos por sala & 232 & 32,28 & 32,0 & 6,47 & $31,44-33,11$ & $30,0-35,0$ \\
\hline
\end{tabular}

Tabela 2 - Distribuição por regiões anatômicas do corpo humano de sintomas osteomusculares, incapacidade funcional, procura por profissional da área de saúde entre os professores da rede municipal de ensino de Natal, RN, 2007.

Table 2 - Distribution of muscle skeletal symptoms, functional disability, demand for professional health care by anatomical regions of the human body, among teachers from the public school system of Natal, RN, Brazil, 2007.

\begin{tabular}{lcccc}
\hline & $\begin{array}{c}\text { Sintomas } \\
\text { nos últimos } \\
12 \text { meses } \\
(\%)\end{array}$ & $\begin{array}{c}\text { Impedimento de realizar } \\
\text { atividades normais por } \\
\text { causa deste problema } \\
\text { nos últimos 12 meses } \\
(\%)\end{array}$ & $\begin{array}{c}\text { Consulta a algum profissional } \\
\text { da área da saúde por causa } \\
\text { desta condição nos últimos } \\
12 \text { meses } \\
(\%)\end{array}$ & $\begin{array}{c}\text { Sintomas } \\
\text { nos últimos } \\
7 \text { dias } \\
(\%)\end{array}$ \\
\hline Pescoço & 53,7 & 17,4 & 20,7 & 24,0 \\
Ombros & 50,4 & 16,5 & 17,8 & 26,4 \\
Parte superior das costas & 58,7 & 15,3 & 17,8 & 28,5 \\
Cotovelos & 11,6 & 4,5 & 4,5 & 5,0 \\
Punhos/ mãos & 51,2 & 17,4 & 16,9 & 22,3 \\
Parte inferior das costas & 53,7 & 22,7 & 18,6 & 26,9 \\
Quadril/ coxas & 25,6 & 10,7 & 10,7 & 11,6 \\
Joelhos & 38,4 & 15,7 & 14,9 & 18,2 \\
Tornozelo/pé & 52,9 & 13,2 & 17,4 & 25,2 \\
\hline
\end{tabular}


consideração o momento da coleta dos dados, independente da região corporal afetada, foi de $63,2 \%$ entre os professores da rede municipal de ensino de Natal, RN. As regiões corporais em que se registrou um maior número de queixas foram a parte superior das costas com $28,5 \%$, parte inferior das costas com $26,9 \%$ e região dos ombros com $26,4 \%$. Na Tabela 2 está descrito detalhadamente o percentual de acometimento em cada região corporal contida no questionário Nórdico.

Com relação à avaliação da qualidade de vida, os domínios que apresentaram menor escore médio foram o meio ambiente, com média de 54,02, e o físico com 62,68. (Tabela 3)

Na comparação dos resultados da avaliação da qualidade de vida entre o grupo de professores que possuíam sintomas osteomusculares e o grupo que não possuíam tal sintomatologia, todos os domínios da qualidade de vida apresentaram diferença estatística significativa, podendo ser observado o maior comprometimento entre os professores com sintomas osteomusculares. (Tabela 4)

\section{Discussão}

Diversos estudos realizados com amostras de professores do ensino básico apresentaram resultados sobre os dados socioeconômicos e ocupacionais (carga horária, tempo de exercício da docência e o número de alunos por sala) semelhantes aos relatados na presente pesquisa, configurando um quadro homogêneo das características do trabalho docente no Brasil ${ }^{11,15,18-21}$.

Com relação específica à predominância de mulheres exercendo a prática da docência no ensino básico, o fato pode ser explicado pelo processo histórico da entrada das mulheres no mercado de trabalho, onde grande parte delas ingressou no campo educacional, sendo a atividade docente rotulada como uma continuidade do trabalho doméstico, assumindo as professoras um papel de "mãe educadora" ${ }^{21}$.

No que diz respeito aos resultados obtidos na avaliação dos domínios da QV, poucos estudos realizados com a população de professores do ensino básico foram encontrados na literatura. Na cidade de Rio Claro,

Tabela 3 - Distribuição dos resultados dos domínios da qualidade de vida dos professores da rede municipal de ensino de Natal, RN, 2007.

Table 3 - Distribution of the results of quality of life domains for teachers from the public school system of Natal, RN, Brazil, 2007.

\begin{tabular}{lcccc}
\hline Domínios da Qualidade de Vida & $\mathrm{n}$ & Média & DP & IC (95\%) \\
\hline Físico & 242 & 62.68 & 16,50 & $60,59-64,77$ \\
Psicológico & 242 & 67,77 & 14,43 & $65,94-69,60$ \\
Relações Sociais & 242 & 68,70 & 17,44 & $66,49-70,91$ \\
Meio Ambiente & 242 & 54,02 & 14,77 & $52,15-55,89$ \\
\hline
\end{tabular}

Tabela 4 - Distribuição dos resultados dos domínios da qualidade de vida entre os professores com e sem sintomatologia osteomuscular da rede municipal de ensino de Natal/RN, 2007

Table 4 - Distribution of the results of quality of life domains among teachers with and without muscle skeletal symptoms from the public school system of Natal, Brazil, 2007.

\begin{tabular}{lcccc}
\hline $\begin{array}{l}\text { Domínios da Qualidade } \\
\text { de Vida }\end{array}$ & $\mathrm{n}$ & $\begin{array}{c}\text { Média dos postos dos } \\
\text { professores com sintomas }\end{array}$ & $\begin{array}{c}\text { Média dos postos dos } \\
\text { professores sem sintomas }\end{array}$ & $p$ \\
\hline Físico & 242 & 103,34 & 148,59 &, $000^{*}$ \\
Psicológico & 242 & 108,20 & 140,25 &, $001^{*}$ \\
Relações Sociais & 242 & 111,49 & 134,61 &, $001^{*}$ \\
Meio Ambiente & 242 & 108,39 & 139,93 &, $001^{*}$ \\
\hline
\end{tabular}

*Diferença estatística significativa em nível de 5\%.

* Statistically significant difference of $5 \%$. 
SP, foram investigados 128 professores de escolas públicas, sendo relatado também maior comprometimento nos domínios físico e meio ambiente ${ }^{15}$. Já na pesquisa desenvolvida com 80 professores do ensino fundamental da cidade de Palotina, PR, o domínio Meio Ambiente também foi o que obteve menor escore médio quando comparado aos demais domínios da qualidade de vida, sendo encontrado valor de 58, $5^{22}$.

Na pesquisa sobre a qualidade de vida desenvolvida com 91 docentes da rede municipal de ensino da cidade de Jequié, BA, também foram descritos resultados que indicavam comprometimento nos domínios limitação por aspecto físico e capacidade funcional. O referido estudo utilizou outro instrumento para avaliar a QV, mas os domínios citados se referem a aspectos avaliados no domínio físico da presente pesquisa ${ }^{23}$.

Outro importante achado na avaliação dos professores da rede municipal de Natal, $\mathrm{RN}$, foi a prevalência de $63,2 \%$ de sintomas osteomusculares, levando em consideração os últimos sete dias em relação ao momento da coleta dos dados, principalmente na região das costas e dos ombros.

Dados semelhantes também apontam uma prevalência de $90,4 \%$ de sintomas osteomusculares entre docentes da rede pública de uma cidade do interior do Estado de São Paulo ${ }^{24}$ e também o maior comprometimento de dor musculoesquelética das regiões corporais dos membros superiores e do dorso, de acordo como o estudo de caráter censitário realizado com 4.496 professores do ensino fundamental na cidade de Salvador, $\mathrm{BA}^{25}$.

Outro estudo sobre as doenças ocupacionais diagnosticadas com maior frequência nos atendimentos realizados a professores pelo Centro de Estudos da Saúde do Trabalhador da cidade de Salvador/BA demonstrou que os distúrbios osteomusculares acometeram a maior parte dos investigados ${ }^{13}$. Na pesquisa realizada com 607 professores da rede municipal de Vitória, ES, 49,26\% dos indivíduos relataram ter necessitado de atendimento médico para problemas com transtornos osteoarticulares ${ }^{18}$.
Nesse contexto apresentado, a prática da docência a longo prazo pode originar diversas patologias músculo-esqueléticas, muitas vezes relacionadas a quadros álgicos intensos, podendo acarretar em absenteís$\operatorname{mos}^{18}$. Tal afirmativa pode fornecer subsídios para o entendimento do considerado percentual de docentes que relataram terem tido impedimentos para realizar atividades de trabalho e atividades domésticas, devido à referida sintomatologia nos últimos 12 meses.

Especificamente sobre o impacto da sintomatologia osteomuscular na qualidade de vida dos investigados, quando comparadas às médias dos domínios da QV dos docentes com e sem sintomas osteomusculares, é evidente o comprometimento do primeiro grupo.

São poucos os estudos encontrados na literatura que avaliaram o impacto dos sintomas osteomusculares na qualidade de vida de professores, principalmente entre os que atuam na educação básica. Apesar de ter sido utilizado outro instrumento diferente do Whoqol-breve para avaliar 157 professores da rede de ensino pública de uma cidade do Estado de São Paulo ${ }^{24}$, praticamente todos os domínios da QV estavam comprometidos nos indivíduos que apresentavam a sintomatologia em questão, destacando-se os aspectos físicos e emocionais, os quais também se apresentaram comprometidos entre os docentes da rede municipal de Natal, RN.

Os fatores avaliados no domínio físico, como a dor, a fadiga e a incapacidade funcional, podem estar geralmente associados à presença de distúrbios do sistema musculoesquelético, o que pode provocar repercussões sobre a qualidade de vida dos indivíduos ${ }^{10}$.

Apesar de não terem sido investigadas questões específicas sobre o uso de medicação entre os participantes da presente pesquisa, uma das questões que compõem o constructo da QV no domínio físico é a dependência de medicação e tratamento, o que possivelmente é um aspecto comum entre indivíduos acometidos pela sintoma- 
tologia osteomuscular, que pode ter contribuído no comprometimento da avaliação do referido domínio da qualidade de vida.

Foi apresentado um maior prejuízo no grupo de docentes com sintomas osteomusculares no que se refere à avaliação do domínio psicológico e da relação social. Ambos os aspectos da qualidade de vida são fatores importantes na manutenção da saúde emocional de um individuo, podendo a resultante dos desequilíbrios desses domínios provocar uma depreciação social e psicológica aos professores ${ }^{4}$.

Já com relação à baixa pontuação do domínio meio ambiente entre os investigados com a sintomatologia osteomuscular, podem-se destacar questões referentes à prática de atividades de lazer e recreação, as quais podem estar comprometidas nos indivíduos com tal quadro patológico, uma vez que a presença de dor e formigamento pode provocar limitação funcional, o que impediria a realização das referidas práticas.

Outra importante questão relacionada à problemática descrita anteriormente é o fato de que praticamente $65 \%$ dos investigados não trabalham no mesmo bairro em que estão localizadas as escolas, tendo muitos deles que se deslocar por grandes distâncias.

Apesar de não ter sido investigada especificamente a estrutura física das escolas municipais de Natal, RN, é possível que as deficiências estruturais existentes nos ambientes das escolas públicas também possam ser um fator de contribuição para o surgimento dos sintomas osteomusculares nos professores, sendo esta uma das questões avaliadas no constructo da qualidade de vida no domínio meio ambiente.

É importante ressaltar que o período de coleta dos dados da presente pesquisa, foi época de grande sobrecarga de trabalho para os professores, mas que faz parte da rotina docente pode ter sido um fator que contribuiu para a alta taxa de sintomas oste- omusculares e para o comprometimento da qualidade de vida desses indivíduos.

Também devem ser consideradas as limitações dos resultados provenientes da utilização de questionários auto-administráveis e de estudos transversais, os quais produzem uma imagem que retratam uma situação de saúde característica de um momento especifico de tempo, avaliando apenas os trabalhadores "sadios" e excluindo aqueles que por ventura estavam afastados das atividades laborais no momento da coleta por motivo de problemas de saúde.

\section{Considerações finais}

Entre os docentes investigados da rede municipal da cidade de Natal, RN, o maior percentual de professores referiu presença da sintomatologia osteomuscular, apresentando o referido grupo comprometimento em todos os domínios da avaliação da qualidade de vida.

A implantação de medidas preventivas que evitem o agravamento da sintomatologia osteomuscular entre a classe de professores investigados é necessária para que não ocorra o afastamento das atividades de trabalho desses profissionais, e o consequente aumento de gastos com tratamentos de saúde e com questões previdenciárias, investimentos esses que poderiam ser mais bem aproveitados na efetivação de políticas de promoção de saúde para esse grupo de trabalhadores.

Agradecimentos: Agradecemos à FAPESB (Fundação de Amparo à Pesquisa do Estado da Bahia) pelo auxílio referente à bolsa de apoio ao curso de doutoramento de Marcos Henrique Fernandes. Ao apoio na coleta dos dados dos discentes do Curso de Fisioterapia da UFRN: Ivanízia Soares, Philippe Manoel, Andressa Gollo, Elida Raquel, Luzianne Juline e Juliana Beatriz. 


\section{Referências}

1. Giovanetti RM. Saúde e apoio social no trabalho: estudo de caso de professores da educação básica pública [dissertação de mestrado]. São Paulo: Universidade de São Paulo; 2006.

2. Brasil. Ministério da Saúde. Doenças relacionadas ao trabalho: manual de procedimentos para os serviços de saúde. Brasília, DF; 2001.

3. Gomes L. Trabalho multifacetado de professores/as: a saúde entre limites [dissertação de mestrado]. Rio de Janeiro: Fundação Oswaldo Cruz - FIOCRUZ. Escola Nacional de Saúde Pública - ENSP; 2002.

4. Libâneo JC. Adeus Professor, Adeus Professora? Novas exigências educacionais e profissão docente. $9^{a}$ Ed. São Paulo: Cortez; 2006.

5. Souza KR, Santos MBM, Pina JÁ, Maria ABV, Carmo MAT, Jensen M. A trajetória do Sindicato Estadual dos Profissionais da Educação do Rio de Janeiro (Sepe-RJ) na luta pela saúde no trabalho. Cienc Saúde Coletiva 2003; 8(4): 1057-68.

6. Santomé JT. O professor em época de neoliberalismo: dimensões sociopolíticos de seu trabalho. In C. Linhares (Org.), Os professores e reinvenção da escola: Brasile Espanha. São Paulo. Cortez; 2001.

7. Minayo MCS, Hartz ZMA, Buss PM. Qualidade de vida e saúde: um debate necessário. Ciênc. Saúde Coletiva 2000; 5(1): 7-18.

8. Alleyne GAO. Health and the quality of life. Rev Panam Salud Publica 2001; 9(1): 1-6.

9. The WHOQOL Group. The World Health Organization quality of life assessment (WHOQOL): position paper from the World Health Organization. Soc Sci Med 1995; 41: 1403-10.

10. Punnett L, Wegman DH. Work-related musculoskeletal disorders: the epidemiologic evidence and the debate. $J$ Electromyogr Kinesiol 2004; 14: 13-23.

11. Carvalho AJFP, Alexandre NMC. Sintomas osteomusculares em professores do ensino fundamental. Rev Bras Fisioter 2006; 10(1): 35-41.

12. Gasparini SM, Barreto SM, Assunção AA. O professor, as condições de trabalho e os efeitos sobre sua saúde. Educação e Pesquisa 2005; 31(2): 189-99.

13. Porto LA, Reis IC, Andrade JM, Nascimento CR, Carvalho FM. Doenças ocupacionais em professores atendidos pelo centro de estudos da saúde do trabalhador (CESAT). Rev Baiana Saúde Pública 2004; 28(1): 33-49.

14. Barros ENC, Alexandre NMC. Cross-cultural adaptation of Nordic musculoskeletal questionnaire. Int Nurs Ver 2003; 50(2): 101-8.
15. Penteado RZ, Pereira IMTB. Qualidade de vida e saúde vocal de professores. Rev Saúde Pública 2007; 41(2): 23643.

16. Fleck MPA, Louzada S, Xavier M, Chachamovich E, Vieira G, Santos L, et al. Aplicação da versão em português do instrumento abreviado de avaliação de qualidade de vida “WHOQOL/breve”. Rev Saúde Pública 2000; 34: 17883.

17. Pinheiro FA, Tróccoli BT, Carvalho CV. Validação do Questionário Nórdico de Sintomas Osteomusculares como medida de morbidade. Rev Saúde Pública 2002; 36(3): 307-12.

18. Marchiori F, Barros MEB, Oliveira SP. Atividade de trabalho e saúde dos professores: o programa de formação como estratégias de intervenção nas escolas. Trabalho, Educação e Saúde 2005; 3(1): 143-70.

19. Neto AMS, Araújo TM, Dutra FRD, Azi GR, Alves RL, Kavalkievicz C et al. Condições de trabalho e saúde de professores da rede particular de ensino de Salvador, Bahia. Rev Baiana Saúde Pública 2000; 24(1/2): 42-56.

20. Delcor NS, Araújo TM, Reis EJFB, Porto LA, Carvalho FM, Silva MO et al. Condições de trabalho e saúde dos professores da rede particular de ensino de Vitória da Conquista, Bahia, Brasil. Cad Saúde Pública 2004; 20(1): 187-96.

21. Codo W. Educação: carinho e trabalho. Petrópolis: Editora Vozes; 1999.

22. Bittencourt MGSQ. Qualidade de vida do professor do ensino fundamental da rede pública municipal de Palotina, PR [dissertação]. Campo Grande: Universidade Católica Dom Bosco; 2005.

23. Fernandes MH, Rocha VM. Qualidade de vida de professores do ensino fundamental: uma perspectiva para a promoção da saúde do trabalhador. J Bras Psiquiatr 2008; 57(1): 23-7.

24. Carvalho AJFP, Alexandre NMC. Qualidade de vida e sintomas osteomusculares relacionados ao trabalho em professores do ensino fundamental. Fisioterapia Brasil 2006; 7(4): 279-84.

25. Cardoso JP, Ribeiro IQB, Araújo TM, Carvalho FM, Reis EJFB. Prevalência de dor musculoesquelética em professores. Rev Bras Epidemiol 2009; 12(4): 604-14.

26. Reis EJFB, Araújo TM, Carvalho FM, Barbalho I, Silva MO. Docência e exaustão emocional. Educ Soc 2006; 27(94): 229-53.

Recebido em: 26/01/10

Versão final reapresentada em: 03/02/11 Aprovado em: 09/02/11 